\title{
Estudo das características somatotípicas, dermatoglíficas e das qualidades físicas de universitários de educação física
}

\author{
Artigo Original
}

\author{
Luís Cláudio Pereira Monteiro \\ Programa de Pós-graduação Stricto Sensu em Ciência da Motricidade Humana da \\ Universidade Castelo Branco / RJ \\ Universidade Estácio de Sá \\ luisclaudiomonteiro@bol,com,br \\ José Fernandes Filho \\ Programa de Pós-graduação Stricto Sensu em Ciência da Motricidade Humana da \\ Universidade Castelo Branco / RJ \\ jff@ism,com,br
}

\author{
Homero da Silva Nahum Júnior \\ Coope UFRJ \\ Universidade Estácio de Sá \\ junior_alema@yahoo,de
}

MONTEIRO, L,C,P; FILHO, J,F,; JUNIOR, H,S,N, Estudo das características somatotípicas, dermatoglíficas e das qualidades físicas de universitários de educação física. Fitness \& Performance Journal, v,3, n,6, p,329-337, 2004,

\begin{abstract}
RESUMO: O presente estudo teve como objetivo verificar características somatotípicas, dermatoglificas e das qualidades físicas de universitários de educação física, A amostra foi constituída por 95 universitários, sendo 40 homens e 55 mulheres com idade estipulada de 20 a 30 anos, cursando até o quarto período, Os procedimentos que foram utilizados na coleta de dados seguiram rigorosamente os procedimentos determinados pelos protocolos de cada teste, Para identificação das características genéticas foi escolhido o protocolo de dermatoglifia de Cumins e Midlo (1942), as medidas de somatotipo foram obtidas pelo método somatotipológico de Heath \& Carter (1990), O teste utilizado para avaliar a resistência aeróbica foi o da corrida de 12 minutos de Cooper (1972); para avaliar a força explosiva, utilizou-se o Sargent Jump Test (1921); para medir a agilidade foi usado o teste Shuttle Rum (1986); o teste de coordenação utilizado foi o de Burppe (1986); e para medir a velocidade foi utilizado o teste de $50 \mathrm{~m}$, A metodologia de tratamento estatístico considerou a abordagem descritiva, através da qual procedemos à obtenção das estimativas para medidas de localização, dispersão e distribuição, Os valores médios e seus desvios padrões, referentes ao somatotipo se apresentaram como: Mulheres: (endomorfia $=5,16 \pm 1,48$, mesomorfia $=3,90 \pm 1,36$ e ectomorfia 2,32 $\pm 1,16$ ), classificação - endomesomórfico, Homens: (endomorfia $=3,05 \pm 1,40$, mesomorfia $=4,70 \pm 1,39$ e ectomorfia 2,20 $\pm 1,18$ ), classificação - mesoendomorfico, Na característica dermatoglifica as fórmulas digitais se distribuíram da seguinte maneira: Homens: (15\% apresentaram ALW, $20 \% 10 \mathrm{~L}, 37 \% \mathrm{~L}>\mathrm{W}, 20 \% \mathrm{~W}>\mathrm{L}$, 2,5\% A > L e 5\% L>A) Mulheres: (5,5\% apresentaram ALW, 12,7 \% 10L, 38,2\%L=W, 23,6\%W > L, 5,5\% A>L, 3,6\% L>A 3,6\% 10w, 5,5\% L=W e 1,8 A=L), Nas qualidades físicas foram apresentados os seguintes resultados: Mulheres: Impulsão vertical 34,73 $\pm 5,68 \mathrm{~cm}$, $\mathrm{Vo2} 34,27 \pm 7,04 \mathrm{ml},(\mathrm{kg}, \mathrm{min})^{-1}$, coordenação $4,9 \pm 0,65$ repetições, velocidade $10,6 \pm 1,13$ Seg e agilidade $12 \pm 1,00 \mathrm{Seg}$, Nos homens, os resultados foram: impulsão vertical, $50,08 \pm 7,18 \mathrm{~cm}$, Vo2 43,12 $\pm 10,04 \mathrm{ml},(\mathrm{kg}, \mathrm{min})^{-1}$, coordenação 5,9 $\pm 0,88 \mathrm{seg}$, velocidade 8,6 $\pm 1,11$ seg e agilidade 10,3 $\pm 0,67 \mathrm{seg}$, Os resultados encontrados mostraram-se dentro das expectativas do estudo, uma vez que o grupo era formado por indivíduos não atletas com características demasiadamente uniformes,
\end{abstract}

Palavras-chave: dermatoglifia, somatotipo, qualidades físicas de universitários de educação física,

\section{Endereço para correspondência:}

Rua Ana Bral, 30 - Vista Alegre - Rio de Janeiro - RJ - CEP 21230-380

Data de Recebimento: outubro / 2004

Data de Aprovação: novembro / 2004

Copyright@ 2008 por Colégio Brasileiro de Atividade Física, Saúde e Esporte.

\begin{tabular}{l|l|}
\hline 3 & 6 \\
\hline
\end{tabular}


A study on somatotypical and dermatoglyphic characteristics and on physical qualities of Physical Education students

The aim of the present study was to verify somatotypes, dermatoglyphics characteristics and the physical qualities of college students, The sample consisted in 95 students: 40 men and 55 female, with ages between 20 and 30 years, attending until the forth period, Procedures used in the collection of data rigorously followed the protocols determined for each test, The protocol of Cumins and Midlo (1942) was chosen to identify the genetic characteristics, The method of Heath and Carter (1968) was used to distinguish somatotypes, A twelve-minute race evaluated the aerobic resistance, the Sargent Jump Test (1921) measured the explosive force and the Shuttle Run Test (1986) observed the individuals agility, Than, a fifty-meter test was used to measure speed, and finally, the Burppe (1986) test calculated coordination, The methodology of statistical treatment had a descriptive approach, through which we proceeded to obtain measurement estimates for localization, dispersion and distribution, The average values and derivates (standard and error deviation) related to somatotypes were: women (endomorphy $=5.16 \pm 1.48$, mesomorphy $=3.90 \pm 1.36$ and ectomorphy $=2.32+1.16$ ); men (endomorphy $=3.05+1.40$, mesomorphy $=4.70 \pm$ 1.39 and ectomorphy $=2.20 \pm 1.18$ ), The digital formulas in the 1.11 seconds and agility $=10.30 .67$ seconds), The results are within the expectations of this study, since the sample was composed of non-athletes presenting homogenic groups, dermatoglyphic characteristics were distributed in the following way: men $15 \%$ presented ALW, 20\% $10 \mathrm{~L}, 37 \% \mathrm{~L}>\mathrm{W}, 20 \% \mathrm{w}>\mathrm{L}, 2.5 \%>\mathrm{L}$ and $5 \% \mathrm{~L}$ $>A$ ); Women (5.5\% presented ALW, $12.7 \% 10 \mathrm{~L}, 38.2 \% \mathrm{I}=\mathrm{w}, 23.6 \% \mathrm{w}>\mathrm{L}, 5.5 \%$ $>\mathrm{L}, 3.6 \% \mathrm{~L}>\mathrm{A}, 3.6 \% 10 \mathrm{w}, 5.5 \% \mathrm{~L}=\mathrm{W}$ and $1.8 \mathrm{~A}=\mathrm{L}$ ), The results regarding physical qualities were: women (vertical impulse $=34.73+5.68 \mathrm{~cm}$, Vo2 $=$ $34.27 \pm 7.04 \mathrm{ml}(\mathrm{Kg}, \mathrm{min})$, coordination $=4.9 \pm 0.65$ repetitions, speed $=$ $10.6 \pm 1.13$ seconds, agility $=12+1.00$ seconds) and men (vertical impulse $=50.08+7.18 \mathrm{~cm}, V_{0} 2=43.12 \pm 10.04 \mathrm{ml}(\mathrm{Kg}, \mathrm{min})$, coordination $=5.9$ +0.88 repetitions, speed $=8.6++$.

Keywords: dermatoglyphics, somatotype, basic physical qualities (VO2 max, agility, speed, coordination and force) of college students of physical education.

\section{INTRODUÇÃO}

As qualidades físicas básicas são muito estudadas em diversas populações (idosos, crianças e universitários), Porém, um grande número de investigações objetiva definir a relação entre a performance dos atletas e as qualidades físicas, principalmente aquelas de maior relevância à modalidade praticada, Glaner (1998) realizou um diagnóstico da aptidão física relacionada à saúde em universitários, observando que estes tinham pouca aptidão física, Já na idade escolar, Matsudo (1990) comparou os níveis de aptidão em diferentes níveis sócio-econômicos, concluindo que não houve diferença,

Fernandes Filho (2003) julga que "o processo de avaliação física $\left(,{ }_{1}\right)$, os resultados obtidos, através da bateria de testes utilizados, são importantes para que se possa desenvolver um bom programa de trabalho físico", Porém, ele também pode ser de grande valia quando associados com outros fatores, Assim, conduziu-se o presente estudo por meio dos mecanismos cognoscitivos que apontam as formas ou estratégias pelas quais o sujeito pode interpretar o seu objeto de estudo, sendo eles: pré-compreensão
Estúdio de las características somatotípicas, Dermatoglíficas y de las calidades físicas del estudiante de Universidade de Educación Física

El presente estudio tuvo como objetivo verificar las características somatotipicas, dermatoglificas y de las calidades físicas de universitários de educación física, La muestra fue constituida por 95 universitarios, siendo 40 hombres y 55 mujeres con edad estipulada de 20 a 30 años cursando hasta el cuarto periodo, los procedimientos que fueron utilizados en la recolecta de datos siguieron rigurosamente los procedimientos determinados por los protocolos de cada prueba, Para identificación de las características genéticas fue escogido el protocolo de dermatoglifia de Cumins y Midlo (1942), las medidas de somatotipo fueron obtenidas por el método somatotipológico de Heath \& Carter (1990), La prueba utilizada para evaluar la resistencia aeróbica fue de la carrera de 12 minutos Cooper (1972), para evaluar la fuerza explosiva se utilizó el Sargent Jump Test, (1921), para medir la agilidad fue usado la prueba shuttle ron (1986), la prueba de coordinación utilizado fue el de Burppe (1986) y para medir la velocidad fue utilizado la prueba de $50 \mathrm{~m}$, La metodología de tratamiento estadístico consideró el abordaje descriptivo, a través de la cual procedimos a la obtención de las estimativas para medidas de localización, dispersión y distribución, Los valores medios y su desviación típica, referentes al somatotipo se hubieron presentado como: Mujeres (endomorfia $=$ $5,16 \pm 1,48$; mesomorfia $=3,90 \pm 1,36$ y ectomorfia $2,32 \pm 1,16)$ Hombres (endomorfia $=3,05 \pm 1,40$; mesomorfia $=4,70 \pm 1,39$ y ectomorfia 2,20 \pm $1,18)$, En la característica dermatoglifica las fórmulas digitales se hubieron distribuido de la siguiente manera: Hombres (15\% presentaron ALW,20\%10L, 37\%L $>\mathrm{W}, 20 \% \mathrm{~W}>\mathrm{L}>2,5 \% \mathrm{~A}>\mathrm{L}$ y $5 \%$; $>\mathrm{A}$ ) Mujeres $(5,5 \%$ presentaron ALW $12,7 \%$ $10 \mathrm{~L}, 38,2 \% \mathrm{~L}=\mathrm{W}, 23,6 \% \mathrm{~W}>\mathrm{L}, 5,5 \% \mathrm{~A}>\mathrm{L}, 3,6 \% \mathrm{~L}>\mathrm{A} 3,6 \% 10 \mathrm{w}, 5,5 \% \mathrm{~L}=\mathrm{W}$ y $1,8 \mathrm{~A}=\mathrm{L}$ ), En las calidades físicas fueron presentados los siguientes resultados: Mujeres: Impulsión vertical excelente 34,73 $\pm 5,68 \mathrm{~cm}, \mathrm{VO} 2_{\text {max }}$ regular $34,27 \pm$ $7,04 \mathrm{ml},(\mathrm{Kg}, \mathrm{min})^{-1}$, coordinación 4,9 $\pm 0,65$ repeticiones, velocidad 10,6 $\pm 1,13$ seg y agilidad $12 \pm 1,00$ seg en los hombres los resultados fueron: impulsión vertical regular para bueno, $50,08 \pm 7,18 \mathrm{~cm}, V_{2 \max }$ regular $43,12 \pm 10,04 \mathrm{ml}(\mathrm{Kg}, \mathrm{min})^{-1}$, coordinación 5,9 $\pm 0,88 \mathrm{seg}$, velocidad $8,6 \pm 1,11 \mathrm{seg}$ y agilidad 10,3 $\pm 0,67$ seg, Los resultados encontrados se hubieron mostrado dentro de las expectativas del estudio, una vez que el grupo era formado por individuos no atletas con características demasiado uniformes,

Palabras clave: dermatoglífia, somatotipo, calidades físicas de los estudiantes de universidad de la educación física. fenomenológica e axiológica e explicação fenomênica, caracterizando-se assim um ciclo dialético e hermenêutico,

Fernandes Filho (1997) afirma que os desenhos formados pelas digito - papilares, na face interna da falangeta dos dedos de ambas as mãos, diferenciavam cada ser humano de seu semelhante; daí se deduz o pressuposto seguro para uma perfeita identificação dos indivíduos, sem possibilidade de erros ou dúvidas, Logo, trata-se de uma marca incontestável, ligada ao genótipo do indivíduo,

Reiterando-se uma das idéias na qual se radica este trabalho, afirma-se que o índice das Impressões Digitais (ID) (FERNANDES FILHO, 1997) estabelece-se já no terceiro mês de vida fetal, juntamente com o sistema nervoso do estrato blasto gênico do ectoderma: as impressões digitais não se alteram durante toda a vida e "incluem o tipo de desenho: a quantidade de linhas nos dedos das mãos",

A maioria dos autores distingue três grupos de desenhos: arco, (A), presilha, (L) e juntamente verticilo e o S-desenho, (W), A 
forma dos desenhos constitui uma características qualitativa, A quantidade de linhas (QL) é o somatório da quantidade total de linhas (SQTL), A quantidade de cristas cutâneas, dentro do desenho, representa a características quantitativa,

Quando observado o comportamento de alguns dos itens avaliados no perfil do atleta de alto rendimento no voleibol (MEDINA, 2000), constata-se que alguns deles se assemelhavam, ratificando a mesma tendência para outras modalidades de jogos, como indicado a seguir: no voleibol de alto rendimento $\operatorname{arco}(A)=0,1 \pm 0,29$, presilha $(L)=6,5 \pm 2,94$, verticilo $(W)$ $=3,4 \pm 2,97$, somatório da quantidade total de linhas (SQTL) $=125,6 \pm 39,12$ e no índice delta $(\mathrm{D} 10)=13,4 \pm 3,11$, ficando evidenciadas diferenciações estabelecidas por particularidades relacionadas a cada um dos dedos e em amplitudes totais; no futsal (DANTAS, 2001) $-\operatorname{arco}(A)=0,0 \pm 0,17$, presilha $(L)=6,6$ \pm 2 , 89, verticilo $(W)=3,5 \pm 2,90$, somatório da quantidade total de linhas $(\mathrm{SQTL})=147,4 \pm 32,88$ e o índice delta (D10) $=13,5 \pm 2,93$,

Segundo Weineck (1991) o treinamento de endurance cardiorrespiratória visa à melhora da capacidade funcional do sistema cardiopulmonar, Um coração mais forte e eficaz é capaz de ejetar tanto no ciclo sistêmico, quanto no ciclo pulmonar maiores quantidades de sangue, em benefício do metabolismo energético e das trocas gasosas, respectivamente, A debilidade deste sistema implica o mau funcionamento do organismo, comprometido em sua totalidade, devido à importância cardiorrespiratória como meio de transporte das funções orgânicas,

Manso (1996) apresenta fatores indicadores de variação da força ao longo da prática esportiva, dividindo-os em quatro grupos principais: fatores biológicos, fatores mecânicos, fatores funcionais e fatores sexuais, Continuando suas considerações, os autores citados, valendo-se de um modelo de terminologia proposta por Vittori (1990) e M, Vélez (1991), acreditam que a manifestação da força se dá de formas diferentes, em função das necessidades de ação, e que sua classificação deve ser sustentada nas causas que provocam a contração muscular, Julgam (DELVILLAR, 1992) imprescindível ao bom desempenho a preocupação com a velocidade de reação, com o deslocamento, com o lançamento e, ainda, com a decisão mental, entre outros aspectos, A rapidez do movimento possui uma estreita relação

Gráfico 1: Todos os itens normatizados - mulheres

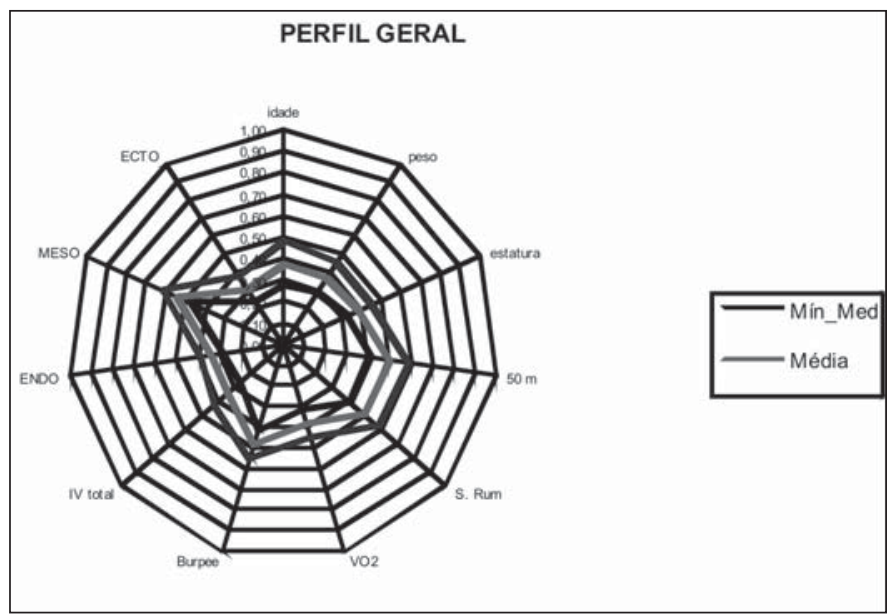

com a velocidade de contração de cada fibra muscular e/ou de grupos musculares,

Dantas (2003) afirma que a agilidade é a "valência física que possibilita mudar a posição do corpo ou a direção do movimento no menor tempo possível", Parece ser a agilidade uma valência física diretamente ligada à velocidade, utilizando como seu conceito basilar a velocidade de deslocamento, a velocidade de reação, a resistência de velocidade, principalmente quanto ao aspecto metabólico, e a velocidade de decisão (MANSO e cols, 1996),

Tubino (1987) crê que a coordenação seja "a qualidade física que permite ao homem assumir a consciência e a execução, levando-o a uma integração progressiva de aquisições, favorecendo-o a uma ação ótima dos diversos grupos musculares na realização de uma seqüência de movimentos com um máximo de eficiência e economia",

Isak (2000) vem demonstrando que o estágio atual da Somatotipologia garante não só a objetividade, mas também a fidedignidade, conforme provam tanto a prática quanto os pressupostos teóricos, baseados no tema, Entre outras aplicações, emprega-se o somatótipo para: descrever e comparar desportistas em distintos níveis; caracterizar as mudanças do físico, durante o crescimento, o envelhecimento e o treinamento; comparar a forma relativa de jogadores masculinos e femininos; além de aplicá-lo como ferramenta de análise da imagem corporal, A análise do somatótipo, é realizada através de medidas antropométricas, como as medidas de estatura, peso corporal, espessura das dobras cutâneas (triciptal, subescapular, supraespinhal e da panturrilha medial), dos diâmetros ósseos de fêmur e úmero, e das circunferências corrigidas de braço e de perna,

\section{OBJETIVO}

O objetivo deste estudo é a busca do conhecimento das características dermatoglíficas e somatotípicas dos alunos universitários e as respectivas distribuições de freqüência com as qualidades físicas básicas (agilidade, velocidade, coordenação, $\mathrm{VO}_{2 \text { máx }} \mathrm{e}$ força), $\bigcirc$ estudo torna-se importante, tendo em vista que, ao conhecermos as características, vamos reunir dados verdadeiros sobre esta população,

Gráfico 2: Todos os itens normatizados - homens

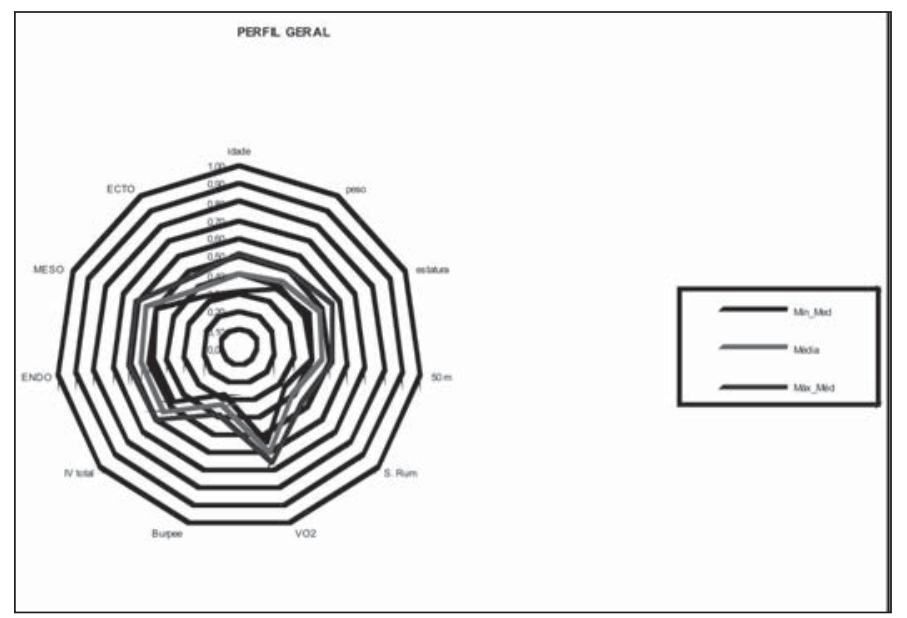


Tabela 1

\section{MULHERES:}

$\operatorname{Arco}(A)=0,55 \pm 1,53$

Presilha $(\mathrm{L})=6,16 \pm 2,90$

Verticilo $(\mathrm{W})=3,29 \pm 3,06$

Somatório da quantidade total de linhas $(S Q T L)=116,69 \pm 46,08$

Índice delta (D10) $=12,75 \pm 3,87$

\section{Tabela 2}

\section{HOMENS:}

$\operatorname{Arco}(A)=0,45 \pm 1,22$

Presilha $(\mathrm{L})=7,05 \pm 2,46$

Verticilo $(\mathrm{W})=2,50 \pm 2,45$

Somatório da quantidade total de linhas (SQTL) $=126,68 \pm 42,73$

Índice delta $(\mathrm{D} 10)=12,05 \pm 2,99$

\section{METODOLOGIA}

\section{Modelo e Tipologia do Estudo}

A presente investigação é um trabalho descritivo com tipologia de perfil,

\section{Amostra}

Utilizou-se um grupo de voluntários formado por universitários de Educação Física, sendo 40 homens e 55 mulheres, com idades entre 23,1 $\pm 3,7$ anos e 24,9 $\pm 3,7$ anos, respectivamente; massa de 75,8 $\pm 11,0 \mathrm{~kg}$ e 29,43 $\pm 8,3 \mathrm{~kg}$, respectivamente; e estatura de $176,2 \pm 8,5 \mathrm{~cm}$ e $162,9 \pm 6,0 \mathrm{~cm}$,

\section{Procedimento da coletas de dados}

Empregou-se o seguinte conjunto de instrumentos para as respectivas aferições:

- Balança - para determinar o peso corporal foi utilizada uma balança da marca Filizola, devidamente calibrada e aferida, com precisão de $100 \mathrm{~g}$ e a escala de 0 a 150 kg,
- Fita métrica - foi utilizada uma fita métrica de metal flexível marca Lufkyn (w606pm) com $200 \mathrm{~cm}$ de comprimento e precisão de $0,1 \mathrm{~cm}$,

- Compasso de dobras cutâneas - para medir a espessura das dobras cutâneas foi utilizado o compasso do tipo Lange, cuja precisão alcança 0,1 mm (Lange skinfold caliper 3006239 fabricado em Maryland),

- Freqüencímetro - para a verificação da freqüência cardíaca, da marca Polar (Polar beat patente ce0537),

- Estadiômetro - portátil da marca Sanny, com 200 cm e divisões em milímetros,

- Paquímetro - avaliamos os diâmetros através de um paquímetro da marca Sanny,

- Quadra de marcação de 20 metros,

Para a obtenção dos dados necessários ao estudo, foram utilizados os seguintes protocolos: o protocolo de dermatoglifia de Cummins e Midlo (1942); somatotipológico de Heath e Carter (ISAK, 2001); velocidade, teste de 50 m (MATSUDO, 1987); força explosiva, teste de impulsão vertical (FERNANDES FILHO, 2003); coordenação, Burpee (MARINS e GIANNICHI, 1996); agilidade, teste de Suttle Run (MATSUDO, 1987); e VO2 máximo (FERNANDES FILHO, 2003),

\section{Tratamento Estatístico}

Empregou-se a análise de freqüência cruzada entre as variáveis dermatoglíficas e as demais, para cada gênero separadamente, visando descrever os grupos através da quantidade de indivíduos em cada classe das respectivas tabelas de freqüência,

\section{Apresentação e Discussão de Resultados}

Os valores médios e seus derivados, referentes aos tipos de desenho, A, L, W e os valores de SQTL e D10 para mulheres e homens se apresentaram da seguinte maneira:

Tabela 3 - Mulheres

\begin{tabular}{llccc}
\hline $\mathrm{VO}_{2}$ máx & Impulsão vertical & Shuttle rum & 50 metros & Burppe \\
\hline $34,27 \pm 7,04 \mathrm{ml},(\mathrm{kg}, \mathrm{min})^{-1}$ & $34,73 \pm 5,68 \mathrm{~cm}$ & $12,01 \pm 1,00 \mathrm{~s}$ & $10,62 \pm 1,13 \mathrm{seg}$ & $4,98 \pm 0,65 \mathrm{rep}$ \\
\hline Tabela 4 - Homens & & & & \\
\hline $\mathrm{VO}_{2}$ máx & Impulsão vertical & Shuttle rum & 50 metros & Burppe \\
\hline $43,12 \pm 10,04 \mathrm{ml},(\mathrm{kg}, \mathrm{min})^{-1}$, & $50,08 \pm 7,18 \mathrm{~cm}$ & $10,31 \pm 0,67 \mathrm{~s}$ & $8,62 \pm 1,11$ seg & $5,95 \pm 0,88$ rep \\
\hline
\end{tabular}

Tabela 5

\begin{tabular}{lcccc}
\hline & ENDOMORFIA & MESOMORFIA & ECTOMORFIA & CLASSIFICAÇÃO \\
\hline MULHERES & $5,16 \pm 1,48$ & $3,90 \pm 1,36$ & $2,32 \pm 1,16$ & ENDOMESOMORFICO \\
HOMENS & $3,05 \pm 1,40$ & $4,70 \pm 1,39$ & $2,20 \pm 1,18$ & MESOENDOMÓRFICO \\
\hline
\end{tabular}

Tabela 6: Teste de 50m (MASCULINO)

\begin{tabular}{lcccrr}
\hline Tempo ' & ALW & LL & L $>W$ & W $>$ L & $18,92 \%(7)$ \\
\hline $6,60-7,61$ & $2,70 \%(1)$ & & $16,22 \%(6)$ & & $35,14 \%(13)$ \\
$7,61-8,62$ & $2,70 \%(1)$ & $16,22 \%(6)$ & $8,11 \%(3)$ & $8,11 \%(3)$ & $21,62 \%(8)$ \\
$8,62-9,63$ & $5,41 \%(2)$ & $5,41 \%(2)$ & $2,70 \%(1)$ & $8,11 \%(3)$ & $24,32 \%(9)$ \\
$9,63-10,64$ & $5,41 \%(2)$ & & $13,51 \%(5)$ & $5,41 \%(2)$ & $100,00 \%(37)$ \\
Total & $16,22 \%(6)$ & $21,62 \%(8)$ & $40,54 \%(15)$ & $21,62 \%(8)$ & \\
\hline
\end{tabular}


Tabela 7: Teste de Suttle Run (MASCULINO)

\begin{tabular}{lccccc}
\hline Tempo ' & ALW & 10L & L > W & W $>$ L & Total \\
\hline $9,06-9,69$ & & & $8,11 \%(3)$ & $8,11 \%(3)$ & $16,22 \%(6)$ \\
$9,69-10,33$ & $5,41 \%(2)$ & $10,81 \%(4)$ & $21,62 \%(8)$ & $2,70 \%(1)$ & $40,54 \%(15)$ \\
$10,33-10,96$ & $5,41 \%(2)$ & $8,11 \%(3)$ & $5,41 \%(2)$ & $5,41 \%(2)$ & $24,32 \%(9)$ \\
$10,96-11,60$ & $5,41 \%(2)$ & $2,70 \%(1)$ & $5,41 \%(2)$ & $5,41 \%(2)$ & $18,92 \%(7)$ \\
Total & $16,22 \%(6)$ & $21,62 \%(8)$ & $40,54 \%(15)$ & $21,62 \%(8)$ & $100,00 \%(37)$ \\
\hline
\end{tabular}

Tabela 8: $\mathrm{VO}_{2}$ max (MASCULINO)

\begin{tabular}{|c|c|c|c|c|c|}
\hline $\mathrm{VO}_{2}(\mathrm{ml}(\mathrm{kg}, \mathrm{min})$ & ALW & $10 \mathrm{~L}$ & $\mathrm{~L}>\mathrm{W}$ & $W>L$ & TOTAL \\
\hline $25,90-34,92$ & $5,41 \%(2)$ & & $5,41 \%(2)$ & & $10,81 \%(4)$ \\
\hline $34,92-43,94$ & $8,11 \%(3)$ & $16,22 \%(6)$ & $21,62 \%(8)$ & $13,51 \%(5)$ & $59,46 \%(22)$ \\
\hline $43,94-52,96$ & $2,70 \%(1)$ & $2,70 \%(1)$ & $8,11 \%(3)$ & $13,51 \%(5)$ & \\
\hline $52,96-61,98$ & $2,70 \%(1)$ & $2,70 \%(1)$ & $5,41 \%(2)$ & & \\
\hline $61,98-71,00$ & & & $5,41 \%(2)$ & & $5,41 \%(2)$ \\
\hline Total & $16,22 \%(6)$ & $21,62 \%(8)$ & $40,54 \%(15)$ & $21,62 \%(8)$ & $100,00 \%(37)$ \\
\hline
\end{tabular}

Tabela 9: Burpee (MASCULINO)

\begin{tabular}{|c|c|c|c|c|c|}
\hline Repetições & ALW & $10 \mathrm{~L}$ & $L>W$ & $W>L$ & Total \\
\hline 4 & $2,70 \%(1)$ & & $2,70 \%(1)$ & & $5,41 \%(2)$ \\
\hline 5 & $5,41 \%(2)$ & $5,41 \%(2)$ & $5,41 \%(2)$ & $5,41 \%(2)$ & $21,62 \%(8)$ \\
\hline 6 & $8,11 \%(3)$ & $10,81 \%(4)$ & $18,92 \%(7)$ & $10,81 \%(4)$ & $48,65 \%(18)$ \\
\hline 7 & & $5,41 \%(2)$ & $10,81 \%(4)$ & & $21,62 \%(8)$ \\
\hline 8 & & & $2,70 \%(1)$ & & $5,41 \%(2)$ \\
\hline Total & $16,22 \%(6)$ & $21,62 \%(8)$ & $40,54 \%(15)$ & $21,62 \%(8)$ & $2,70 \%(1)$ \\
\hline
\end{tabular}

Tabela 10: IV Total (MASCULINO)

\begin{tabular}{lccccc}
\hline Imp, vert $(\mathrm{cm})$ & ALW & 10L & $\mathrm{L}>$ W & W $>$ L & Total \\
\hline $40,0-45,8$ & $10,81 \%(4)$ & $2,70 \%(1)$ & $10,81 \%(4)$ & $8,11 \%(3)$ & $32,43 \%(12)$ \\
$45,8-51,6$ & $2,70 \%(1)$ & $5,41 \%(2)$ & $16,22 \%(6)$ & $2,70 \%(1)$ & $27,03 \%(10)$ \\
$51,6-57,4$ & $2,70 \%(1)$ & $10,81 \%(4)$ & $5,41 \%(2)$ & $5,41 \%(2)$ & $24,32 \%(9)$ \\
$57,4-63,2$ & & $2,70 \%(1)$ & $2,70 \%(1)$ & $5,41 \%(2)$ & $10,81 \%(4)$ \\
$63,2-69,0$ & & & $5,41 \%(2)$ & $5,41 \%(2)$ & $100,00 \%(37)$ \\
Total & $16,22 \%(6)$ & $21,62 \%(8)$ & $40,54 \%(15)$ & $21,62 \%(8)$ & \\
\hline
\end{tabular}

Tabela 11: Endomorfia (MASCULINO)

\begin{tabular}{lccccc}
\hline Classe & ALW & $10 \mathrm{~L}$ & $\mathrm{~L}>\mathrm{W}$ & $\mathrm{W}>\mathrm{L}$ & Total \\
\hline $0,3-1,94$ & & $5,41 \%(2)$ & $8,11 \%(3)$ & $5,41 \%(2)$ & $18,92 \%(7)$ \\
$1,94-3,58$ & $13,51 \%(5)$ & $8,11 \%(3)$ & $18,92 \%(7)$ & $2,70 \%(1)$ & $43,24 \%(16)$ \\
$3,58-5,22$ & $2,70 \%(1)$ & $8,11 \%(3)$ & $10,81 \%(4)$ & $13,51 \%(5)$ & $35,14 \%(13)$ \\
$5,22-6,86$ & & & & & \\
$6,86-8,50$ & & & $2,70 \%(1)$ & & $2,70 \%(1)$ \\
Total & $16,22 \%(6)$ & $21,62 \%(8)$ & $40,54 \%(15)$ & $21,62 \%(8)$ & $100,00 \%(37)$ \\
\hline
\end{tabular}

Tabela 12: Mesomorfia (MASCULINO)

\begin{tabular}{lccccc}
\hline Classe & ALW & IOL & L > W & W $>$ L & Total \\
\hline $1,30-2,85$ & & $2,70 \%(1)$ & & $2,70 \%(1)$ & $5,41 \%(2)$ \\
$2,85-4,40$ & $5,41 \%(2)$ & $8,11 \%(3)$ & $13,51 \%(5)$ & $5,41 \%(2)$ & $32,43 \%(12)$ \\
$4,40-5,95$ & $8,11 \%(3)$ & $8,11 \%(3)$ & $18,92 \%(7)$ & $10,81 \%(4)$ & $45,95 \%(17)$ \\
$5,95-7,50$ & $2,70 \%(1)$ & $2,70 \%(1)$ & $8,11 \%(3)$ & $2,70 \%(1)$ & $16,22 \%(6)$ \\
Total & $16,22 \%(6)$ & $21,62 \%(8)$ & $40,54 \%(15)$ & $21,62 \%(8)$ & $100,00 \%(37)$ \\
\hline
\end{tabular}

Tabela 13: Ectomorfia (MASCULINO)

\begin{tabular}{lccccc}
\hline Classe & ALW & $10 \mathrm{~L}$ & $\mathrm{~L}>\mathrm{W}$ & $\mathrm{W}>\mathrm{L}$ & Total \\
\hline $0,60-1,64$ & $8,11 \%(3)$ & $10,81 \%(4)$ & $13,51 \%(5)$ & $10,81 \%(4)$ & $43,24 \%(16)$ \\
$1,64-2,68$ & $5,41 \%(2)$ & & $13,51 \%(5)$ & $2,70 \%(1)$ & $21,62 \%(8)$ \\
$2,68-3,72$ & & $8,11 \%(3)$ & $10,81 \%(4)$ & $5,41 \%(2)$ & $24,32 \%(9)$ \\
$3,72-4,76$ & $2,70 \%(1)$ & & & $2,70 \%(1)$ & $5,41 \%(2)$ \\
$4,76-5,80$ & & $2,70 \%(1)$ & $2,70 \%(1)$ & & $5,41 \%(2)$ \\
Total & $16,22 \%(6)$ & $21,62 \%(8)$ & $40,54 \%(15)$ & $21,62 \%(8)$ & $100,00 \%(37)$ \\
\hline
\end{tabular}


Ao observar-se a literatura, percebe-se que os resultados encontrados diferem dos padrões para desportos de jogos com resistência de velocidade, o alto nível de D10, a falta de arco (A), o aumento da parcela de (W) e o aumento do SQTL caracterizam modalidades esportivas,

Esta diferença era esperada, uma vez que o grupo estudado não era de atletas e sim de universitários,

Guba e Tchernova (1995), citados por Fernandes Filho, 1997), observam que a complexidade dos desenhos indicaria marcas, de prognóstico da compleição definitiva, e o aumento de linhas, ao contrário, refere-se ao desenvolvimento das qualidades de velocidade e de força,

Em função dos resultados observados no nosso estudo, observouse que o número delta (D10) foi menor do que os encontrados nos atletas de alto rendimento, o que era de se esperar, pois a literatura mostra que um alto índice delta é uma característica de atletas,

Os valores médios e seus derivados, referentes à aptidão física apresentaram:

Analisando os resultados obtidos quanto às qualidades físicas observamos que, no teste de impulsão vertical, as mulheres apresentaram valores excelentes em relação à tabela de desempenho; já os homens apresentaram valores de regular para bom, $\bigcirc$ resultado obtido no teste de 12 minutos em homens e mulheres foi considerado regular de acordo com a tabela de desempenho, apresentando um desvio padrão de 7,04 para mulheres e de 10,04 para homens, $\bigcirc$ teste de coordenação apresentou uma média de 4,9 repetições e um desvio padrão de 0,65 para mulheres e 5,9 repetições com um desvio padrão de 0,88 para homens, $\bigcirc$ teste de velocidade apresentou uma média de 10,6 s com um desvio padrão de 1,13 para mulheres e 8,6 s e desvio padrão de 1,11 para homens, $\bigcirc$ teste de agilidade apresentou uma média de 12 s com um desvio padrão de 1,00 para mulheres e 10,3 s com desvio padrão de 0,67 para homens,

Mancilla (2001), no Chile, correlacionou as características dermatoglíficas com qualidades físicas de alunos do primeiro ano de pedagogia da Universidade de Tarapacá, tendo como resultado uma alta correlação, tanto em homens, como em mulheres,

Os resultados obtidos se mostraram dentro da normalidade para a população estudada,

Os valores médios e seus derivados, referentes ao somatótipo, apresentaram-se como:

$\mathrm{L}>\mathrm{W}(16,22 \%)$ e $10 \mathrm{~L}(16,22 \%)$ do grupo avaliado obtiveram as primeiras classificações no teste de 50 metros, com o tempo de $(6,6-7,6)$ para $L>W$ e $(7,6-8,6)$ para $10 L$, demonstrando não só que dentro das vias energéticas e metabólicas o tipo de I $D(L)$ presilha é predominante nesta prova, corroborando Nikitiuk

Tabela 14: Teste de 50m (FEMININO)

\begin{tabular}{|c|c|c|c|c|c|c|c|}
\hline Velocidade $\mathrm{T}^{\prime}$ & ALW & $10 \mathrm{~L}$ & $L>W$ & $W>L$ & $10 \mathrm{~W}$ & $\mathrm{~L}=\mathrm{W}$ & Total \\
\hline $8,17-9,49$ & & & $10,20 \%(5)$ & $2,04 \%(1)$ & & $2,04 \%(1)$ & $14,29 \%(7)$ \\
\hline $9,49-10,81$ & & $2,04 \%(1)$ & $22,45 \%(11)$ & $12,24 \%(6)$ & $4,08 \%(2)$ & $2,04 \%(1)$ & $42,86 \%(21)$ \\
\hline $10,81-12,13$ & $4,08 \%(2)$ & $10,20 \%(5)$ & $6,12 \%(3)$ & $12,24 \%(6)$ & & $2,04 \%(1)$ & $34,69 \%(17)$ \\
\hline $12,13-13,45$ & $2,04 \%(1)$ & $2,04 \%(1)$ & $4,08 \%(2)$ & & & & $8,16 \%(4)$ \\
\hline Total & $6,12 \%(3)$ & $14,29 \&(7)$ & $42,86 \%(21)$ & $26,53 \%(13)$ & $4,08 \%(2)$ & $6,12 \%(3)$ & $100,00 \%(49)$ \\
\hline
\end{tabular}

Tabela 15: Teste de Suttle Run (FEMININO)

\begin{tabular}{|c|c|c|c|c|c|c|c|}
\hline Velocidade $\mathrm{T}^{\prime}$ & ALW & $10 \mathrm{~L}$ & $\mathrm{~L}>\mathrm{W}$ & $W>L$ & $10 \mathrm{~W}$ & $\mathrm{~L}=\mathrm{W}$ & Total \\
\hline $10,41-11,22$ & $2,04 \%(1)$ & $14,29 \%(7)$ & $8,16 \%(4)$ & $2,04 \%(1)$ & & $26,53 \%(13)$ & \\
\hline $11,22-12,04$ & $2,04 \%(1)$ & $2,04 \%(1)$ & $16,33 \%(8)$ & $4,08 \%(2)$ & $2,04 \%(1)$ & $6,12 \%(3)$ & $32,66 \%(16)$ \\
\hline $12,04-12,86$ & $2,04 \%(1)$ & $6,12 \%(3)$ & $4,08 \%(2)$ & $8,16 \%(4)$ & & & $20,41 \%(10)$ \\
\hline $12,86-13,68$ & $2,04 \%(1)$ & $4,08 \%(2)$ & $4,08 \%(2)$ & $6,12 \%(3)$ & & & $16,33 \%(8)$ \\
\hline $13,68-14,50$ & & $4,08 \%(2)$ & & & & $4,08 \%(2$ & \\
\hline Total & $6,12 \%(3)$ & $14,29 \&(7)$ & $42,86 \%(21)$ & $26,53 \%(13)$ & $4,08 \%(2)$ & $6,12 \%(3)$ & $100,00 \%(49)$ \\
\hline
\end{tabular}

Tabela 16: $\mathrm{VO}_{2} \max$ (FEMININO)

\begin{tabular}{|c|c|c|c|c|c|c|c|}
\hline $\mathrm{VO}_{2}(\mathrm{ml}(\mathrm{kg}, \mathrm{min})$ & ALW & $10 \mathrm{~L}$ & $L>W$ & $W>L$ & $10 \mathrm{~W}$ & $\mathrm{~L}=\mathrm{W}$ & Total \\
\hline $13,10-21,97$ & & & $2,04 \%(1)$ & $2,04 \%(1)$ & & & $4,08 \%(2)$ \\
\hline $21,97-30,84$ & $2,04 \%(1)$ & $2,04 \%(1)$ & $12,24 \%(6)$ & $12,24 \%(6)$ & & $2,04 \%(1)$ & $30,61 \%(15)$ \\
\hline $30,84-39,71$ & $4,08 \%(2)$ & $12,24 \%(6)$ & $16,33 \%(8)$ & $8,16 \%(4)$ & $4,08 \%(2)$ & $2,04 \%(1)$ & $46,94 \%(23)$ \\
\hline $39,71-48,58$ & & & $12,24 \%(6)$ & $4,08 \%(2)$ & & $2,04 \%(1)$ & $18,37 \%(9)$ \\
\hline Total & $6,12 \%(3)$ & $14,29 \%(7)$ & $42,86 \%(21)$ & $26,53 \%(13)$ & $4,08 \%(2)$ & $6,12 \%(3)$ & $100,00 \%$ (49 \\
\hline
\end{tabular}

Tabela 17: Burpee (FEMININO)

\begin{tabular}{|c|c|c|c|c|c|c|c|}
\hline repetições & ALW & $10 \mathrm{~L}$ & $\mathrm{~L}>\mathrm{W}$ & $W>L$ & $10 \mathrm{~W}$ & $L=W$ & Total \\
\hline 4 & $2,04 \%(1)$ & $4,08 \%(2)$ & $8,16 \%(4)$ & $4,08 \%(2)$ & & $2,04 \%(1)$ & $20,41 \%(10)$ \\
\hline 5 & $4,08 \%(2)$ & $10,20 \%(5)$ & $18,37 \%(9)$ & $22,45 \%(11)$ & $4,08 \%(2)$ & $4,08 \%(2)$ & $63,26 \%(31)$ \\
\hline 6 & & & $16,33 \%(8)$ & & & & $16,33 \%(8)$ \\
\hline \multicolumn{8}{|l|}{7} \\
\hline Total & $6,12 \%(3)$ & $14,29 \&(7)$ & $42,86 \%(21)$ & $26,53 \%(13)$ & $4,08 \%(2)$ & $6,12 \%(3)$ & $100,00 \%(49)$ \\
\hline
\end{tabular}


(2001), como também que, no resultado geral, ( $\mathrm{L}>\mathrm{W})$ 40,54\% confirma a velocidade do grupo,

A tabela 7 demonstra que $21,62 \%$ de $L>W$, registra tempo de 9,6 - 10,3 seg, mais uma vez confirmando a velocidade como fator predominante no tipo de I D (L) presilha e indicando que a distribuição das outras formas digitais oscila entre $5,4 \%$ e 10,81\%,

40,54\% da amostra obteve índices de velocidade entre 9,69 e 10,33 Seg,

Na tabela 8, 59,46\% do grupo fica entre 34,92\% e 43,94\% de VO2 máximo e percebe-se ainda um equilíbrio entre $L>$ $W(21,62 \%)$ e $10 L(16,22)$, mas nota-se uma elevação na fórmula digital $W>L(13,51 \%)$, Mesmo assim, o grupo analisado fica entre os valores médios de VO2 máximo (FERNANDES FILHO, 2003),

Na tabela 9 observam-se fórmulas digitais na seguinte ordem: $\mathrm{L}>\mathrm{W}(18,92 \%), 10 \mathrm{~L}(10,81 \%)$ e $\mathrm{W}>\mathrm{L}(10,81 \%)$, totalizando $48,65 \%$ do grupo avaliado com 6 repetições no referido teste, que avalia as qualidades físicas coordenação e agilidade (MARINS e GIANNICHI, 1996) e mostra um aumento na I D (W) verticilo,
Nesta tabela observa-se um equilíbrio nas fórmulas digitais, Nota-se ainda que os resultados obtidos apresentaram uma maior variação, mas mesmo assim os valores foram de médio para alto (40 a 63,2 cm), conforme Fernandes Filho (2003), indicando novamente o predomínio da I D (L) presilha (NIKITIUK, 2001),

A tabela 11 mostra que 43,24\% do grupo tem o componente endomórfico $(1,94$ - 3,58) que, segundo Cárter (1980), é considerado de baixo para moderado; outro grupo $(37,84 \%)$ tem o componente, apresentando valores de 3,58 a 8,50, considerados altos e com riscos para saúde.

Esta tabela mostra que quase $80 \%$ do grupo apresenta o componente mesomorfo entre 2,85 e 5,95, considerado de moderado a alto por Carter (1980), caracterizando este grupo como possuidor de moderado a alto desenvolvimento músculo-esquelético: diâmetros ósseos de maior a grande volume e articulações de maiores a grandes dimensões,

A tabela 13 mostra que 43,24\% do grupo apresenta o componente ectomorfo entre 0,60 e 1,64, considerado baixo por Carter (1980), com linearidade relativa de grande volume por unidade de altura e extremidades relativamente volumosas, 45,64\% do grupo apresenta o componente ectomorfo de baixo a moderado: $1,64-3,72$,

Tabela 18: IV Total (FEMININO)

\begin{tabular}{|c|c|c|c|c|c|c|c|}
\hline Imp, vert (cm) & ALW & $10 \mathrm{~L}$ & $L>W$ & $W>L$ & $10 \mathrm{~W}$ & $\mathrm{~L}=\mathrm{W}$ & Total \\
\hline $22-28$ & & & $6,12 \%(3)$ & $4,08 \%(2)$ & & & $10,20 \%(5)$ \\
\hline $28-34$ & $4,08 \%(2)$ & $6,12 \%(3)$ & $10,20 \%(5)$ & $6,12 \%(3)$ & & $2,04 \%(1)$ & $28,57 \%(14)$ \\
\hline $34-40$ & $2,04 \%(1)$ & $8,16 \%(4)$ & $14,29 \%(7)$ & $10,20 \%(5)$ & $4,08 \%(2)$ & $2,04 \%(1)$ & $40,82 \%(20)$ \\
\hline $40-46$ & & & $12,24 \%(6)$ & $6,12 \%(3)$ & & $2,04 \%(1)$ & $20,41 \%(10)$ \\
\hline Total & $6,12 \%(3)$ & $14,29 \&(7)$ & $42,86 \%(21)$ & $26,53 \%(13)$ & $4,08 \%(2)$ & $6,12 \%(3)$ & $100,00 \%(49)$ \\
\hline
\end{tabular}

Tabela 19: Endomorfia (FEMININO)

\begin{tabular}{|c|c|c|c|c|c|c|c|}
\hline Classe & ALW & $10 \mathrm{~L}$ & $\mathrm{~L}>\mathrm{W}$ & $W>L$ & $10 \mathrm{~W}$ & $\mathrm{~L}=\mathrm{W}$ & Total \\
\hline $2,10-3,24$ & & & $10,20 \%(5)$ & $2,04 \%(1)$ & $2,04 \%(1)$ & & $14,29 \%(7)$ \\
\hline $3,24-4,38$ & & & $6,12 \%(3)$ & $2,04 \%(1)$ & & $4,08 \%(2)$ & $12,24 \%(6)$ \\
\hline $4,38-5,52$ & $4,08 \%(2)$ & $4,08 \%(2)$ & $8,16 \%(4)$ & $8,16 \%(4)$ & & & $24,49 \%(12)$ \\
\hline $5,52-6,66$ & $2,04 \%(1)$ & $10,20 \%(5)$ & $10,20 \%(5)$ & $6,12 \%(3)$ & $2,04 \%(1)$ & $2,04 \%(1)$ & $32,66 \%(16)$ \\
\hline $6,66-7,80$ & & & $8,16 \%(4)$ & $8,16 \%(4)$ & & & $16,33 \%(8)$ \\
\hline Total & $6,12 \%(3)$ & $14,29 \&(7)$ & $42,86 \%(21)$ & $26,53 \%(13)$ & $4,08 \%(2)$ & $6,12 \%(3)$ & $100,00 \%(49)$ \\
\hline
\end{tabular}

Tabela 20: Mesomorfia (FEMININO)

\begin{tabular}{|c|c|c|c|c|c|c|c|}
\hline Classe & ALW & $10 \mathrm{~L}$ & $\mathrm{~L}>\mathrm{W}$ & $W>L$ & $10 \mathrm{~W}$ & $\mathrm{~L}=\mathrm{W}$ & Total \\
\hline $0,50-1,72$ & & & $2,04 \%(1)$ & $4,08 \%(2)$ & & & $6,12 \%(3)$ \\
\hline $1,72-2,94$ & & $2,04 \%(1)$ & $14,29 \%(7)$ & $2,04 \%(1)$ & $2,04 \%(1)$ & $2,04 \%(1)$ & $22,45 \%(11)$ \\
\hline $2,94-4,16$ & $4,08 \%(2)$ & $4,08 \%(2)$ & $12,24 \%(6)$ & & & & $20,41 \%(10)$ \\
\hline $4,16-5,38$ & $2,04 \%(1)$ & $6,12 \%(3)$ & $10,20 \%(5)$ & $12,24 \%(6)$ & $2,04 \%(1)$ & $4,08 \%(2)$ & $36,73 \%(18)$ \\
\hline $5,38-6,60$ & & $2,04 \%(1)$ & $4,08 \%(2)$ & $8,16 \%(4)$ & & & $14,29 \%(7)$ \\
\hline Total & $6,12 \%(3)$ & $14,29 \&(7)$ & $42,86 \%(21)$ & $26,53 \%(13)$ & $4,08 \%(2)$ & $6,12 \%(3)$ & $100,00 \%(49)$ \\
\hline
\end{tabular}

Tabela 21 : Ectomorfia (FEMININO)

\begin{tabular}{|c|c|c|c|c|c|c|c|}
\hline Classe & ALW & $10 \mathrm{~L}$ & $\mathrm{~L}>\mathrm{W}$ & $W>L$ & $10 \mathrm{~W}$ & $\mathrm{~L}=\mathrm{W}$ & Total \\
\hline $0,30-1,32$ & & $4,08 \%(2)$ & $4,08 \%(2)$ & $12,24 \%(6)$ & & & $20,41 \%(10)$ \\
\hline $1,32-2,34$ & & $4,08 \%(2)$ & $18,18 \%(10)$ & $6,12 \%(3)$ & $4,08 \%(2)$ & $2,04 \%(1)$ & $36,73 \%(18)$ \\
\hline $2,34-3,36$ & $6,12 \%(3)$ & $4,08 \%(2)$ & $6,12 \%(3)$ & $6,12 \%(3)$ & & $2,04 \%(1)$ & $24,49 \%(12)$ \\
\hline $3,36-4,38$ & & $2,04 \%(1)$ & $10,20 \%(5)$ & $2,04 \%(1)$ & & $2,04 \%(1)$ & $16,33 \%(8)$ \\
\hline $4,38-5,40$ & & & $2,04 \%(1)$ & & & & $2,04 \%(1)$ \\
\hline Total & $6,12 \%(3)$ & $14,29 \&(7)$ & $42,86 \%(21)$ & $26,53 \%(13)$ & $4,08 \%(2)$ & $6,12 \%(3)$ & $100,00 \%(49)$ \\
\hline
\end{tabular}


Esta tabela mostra um predomínio de $\mathrm{L}>\mathrm{W}$ nos melhores resultados, com 10,20\% entre os tempos de 8,17 a 9,49 seg e $22,45 \%$ entre os tempos de 9,49 a 10,81 seg, demonstrando assim que dentro das vias energéticas e metabólicas o tipo de I D (L) presilha é predominante nesta prova de velocidade (NIKITIUK, 2001),

No resultado geral $L>W, 40,54 \%$ confirma a velocidade do grupo avaliado.

A tabela 15 demonstra que $30,62 \%$ de $L>W$ registra tempo entre 10,41 e 12,04 seg, confirmando a velocidade como fator predominante no tipo de I D (L) e indicando que a distribuição das outras formas digitais oscila entre 2,04\% e 6,12\%,

$59,19 \%$ da amostra obtiveram a velocidade entre 10,41 e 12,04 seg,

Nesta tabela 46,94\% do grupo fica entre 30,84 e 39,71 de VO2 máximo e percebe-se ainda um equilíbrio entre $L>W(16,33 \%)$, $10 \mathrm{~L}(12,24 \%)$ e $W>L(8,16 \%)$, Assim, o grupo analisado fica entre os valores médios de VO2 máximo (FERNANDES FILHO, 2003),

Na tabela 17, observam-se fórmulas digitais na seguinte ordem: $\mathrm{W}>\mathrm{L}(22,45 \%), \mathrm{L}>\mathrm{W}(18,37 \%), 10 \mathrm{~L}(10,20 \%), \operatorname{ALW}(4,08 \%)$ e $\mathrm{L}=\mathrm{W}(4,08 \%)$, totalizando $63,26 \%$ do grupo avaliado com 5 repetições no referido teste, que avalia as qualidades físicas coordenação e agilidade (MARINS e GIANNICHI, 1996) e mostra um aumento na I D (W) verticilo,

Nesta tabela observa-se um equilíbrio nas fórmulas digitais, Notase ainda que os resultados obtidos apresentaram maior variação, $\mathrm{L}>\mathrm{W}(14,29 \%), \mathrm{W}>\mathrm{L}(10,20 \%), 10 \mathrm{~L}(8,16 \%), 10 \mathrm{~W}(4,08 \%)$ e ALW (2,04\%), totalizando $40,82 \%$ do grupo avaliado com o resultado de 34 a $40 \mathrm{~cm}$, Mesmo assim, houve um predomínio da I D (L) presilha, ,

Na tabela 19, 32,66\% do grupo tem o componente endomórfico classe $(5,52-6,66)$ e 24,49\%, (4,38 - 5,52), totalizando $57,15 \%$ do grupo avaliado, o que, segundo Carter (1980), é considerado moderado com moderada adiposidade relativa: gordura subcutânea cobre os contornos musculares e ósseos; aparência mais branda,

Esta tabela mostra que $36,73 \%$ do grupo apresenta o componente mesomorfo entre 4, 16 a 5,38, considerado moderado por Carter (1980), apresentando como característica um moderado desenvolvimento músculo-esquelético relativo: maior volume muscular e ósseo e articulações de maiores dimensões, $42,86 \%$ do grupo apresenta o componente mesomorfo entre 1,72 a 4,16, considerado de baixo a moderado por Carter (1980),

A tabela 21 apresenta 36,73\% do grupo com o componente ectomorfo entre 1,32 a 2, 34, considerado baixo por Carter (1980), com característica de linearidade relativa de grande volume por unidade de altura e extremidades relativamente volumosas; $24,49 \%$ apresenta componente ectomorfo entre 2,34 a 3,36, $20,41 \%$ entre 0,30 a 1,32 e $16,33 \%$ entre 3,36 a 4,38,

\section{Considerações gerais e recomendações}

O presente estudo foi realizado em estudantes de Educação Física que cursavam até o quarto período, Concluímos que a aptidão física destes não se encontrava muito boa, mostrando que, apesar de serem estudantes da área da saúde, o grupo não apresentou uma preocupação muito grande com a própria aptidão,

Ao observar a literatura percebemos que os resultados encontrados diferem dos padrões para desportos de jogos e resistência de velocidade, o alto nível de D10, a falta de arco (A), o aumento da parcela de W e o aumento do SQTL caracterizam modalidades esportivas, No nosso estudo, observou-se que o número delta (D10) foi menor do que os encontrados nos atletas de alto rendimento e que o componente endomórfico foi maior do que os apresentados nos estudos semelhantes,

Os resultados encontrados mostraram-se dentro das expectativas do estudo, uma vez que o grupo era formado por indivíduos não atletas, não sedentários e com características demasiadamente uniformes, ou seja, baixa variabilidade, Isto pode ser um indício de que a dermatoglifia se aplica tanto para populações de atletas, como para as de não atletas,

Concluímos que o resultado do estudo responde à necessidade de comprovação do problema apresentado: o conhecimento das características somatotípicas e dermatoglíficas e das qualidades físicas,

Recomenda-se que sejam realizadas novas investigações sobre o tema, aumentando a amostra, ou um estudo considerando outra faixa etária, permitindo assim um melhor conhecimento do assunto, Sugere-se também, uma investigação do tipo longitudinal,

Ressalta-se que esta pesquisa também poderia ser realizada em grupos diferentes do estudo, o que, provavelmente, traria uma visão mais ampla da abrangência temporal dos fenômenos, como também ocorreria se houvesse uma investigação em atletas de alto rendimento de diversos desportos,

A investigação deste estudo não deve ficar restrita apenas à identificação dos perfis, mas deve, sim, considerar a correlação entre as diversas variáveis estudadas, no que diz respeito às qualidades físicas, com outras existentes e também com outras características antropométricas,

Reconhecendo-se a importância da dermatoglifia como uma das variáveis para a orientação e a seleção de talentos, tornase fundamental a obtenção deste perfil em outros estudos de identificação de características do ser humano.

\section{REFERÊNCIAS BIBLIOGRÁFICAS}

ABRAMOVAT, $F_{;} ;$JDANOVA A, $G_{i}$ NIKITINA T,M, Impressões Dermatoglíficas e Somatotipo - marcas de constituição de diferentes níveis // Atualidades médicas e antropológicas nos esportes, Moscou, 1990,- cap,2, -pág, 94-95,

COOPER, Kenneth H, Aptidáo física em qualquer idade, Rio de Janeiro: Fórum 1972,

CUMMINS H.; MIDLO C,H. Palmar and plantar dermatoglyphics in primates,- Philadelphia, 1942, 257p,

DANTAS, E, H, M, A prática da preparação física, Rio de Janeiro: Shape, 2003,

DANTAS, P, M, S, Identificação dos perfis, genético da aptidão física e somatotípico que caracterizam atletas masculinos, de alto rendimento, participantes do futsal adulto, no Brasil, 2000, Dissertação de mestrado em Ciência da Motricidade Humana, Universidade Castelo Branco, Rio de Janeiro,

DEL VILLAR, C, A, La preparación fisica del futbol basada en el atletismo, Madrid - España: Gymnos, 1992,

FERNANDES FILHO, J, A prática da avaliação física, Rio de Janeiro: SHAPE, 2003, 
Impressões dermatoglíficas - marcas genéticas na seleção dos tipos de esporte e lutas (a exemplo de desportista do Brasil), Tese de Doutorado, Moscou, URSS, 1997

GLANER, M,F, Diagnóstico da aptidão física relacionada à saúde de universitários, Revisto Brasileira de Atividade Física e Saúde, Volume 3 número 4-1998,

MANCILLA,L,A, Caracteristicas dermatoglificas y cualidades físicas de los alumnos de primer año de la carrera de pedagogía en educación física de la universidade de Tarapacá, Dissertação, Universidad de Tarapacá, Chile, 2001,

MANSO,J,M, G, VALDIVIELSO,M,N, \& CABALLERO,J,A,R, Pruebas para la valoración de la capacidade motriz em el desporte, Madrid:Gyminos, 1996,

MARINS, João C, B, \& GIANNICHI, Ronaldo S, Avaliação e prescrição de atividade física guia prático, Rio de Janeiro: SHAPE, 1996,

MATSUDO, V,K,R, Testes em ciências do esporte, $4^{a}$ ed, $S, C$, do Sul: Gráficos Burti,1987,

MATSUDO, V,K e FERREIRA, M, Comparação da aptidão física de escolares de Itaquera e São Caetano do Sul Revista Brasileira Ciência Movimento, Abril, 1990
MEDINA, M, F, Identificaçáo dos perfis genéticos e somatotípico que caracterizam atletas de voleibol masculino adulto de alto rendimento no Brasil, 2000, Dissertação de Mestrado em Ciência da Motricidade Humana, Universidade Castelo Branco, Rio de Janeiro

NIKITIUK B,A, Constituição humana // Novidades e técnicas científicas: Série Antropologia,- Moscou, 1991,Tomus 4, pág,152,

Impressões Dermatoglíficas como marcas do desenvolvimento pré-natal do ectoderma // Marcas genéticas na antropologia e medicina: Anais de trabalhos científicos no Simpósio, Rimelnitzki,

1988, pág, 133,

SOCIEDADE INTERNACIONAL PARA AVANÇO DA CINEANTROPOMETRIA - ISAK, Apostila de curso, Rio de Janeiro, 2000,

THOMAS,R,J \& NELSON,K,J, Métodos de pesquisa em atividade física $3^{a}$ ed, Porto Alegre: Artmed, 2002,

TUBINO, M, G Metodologia científica do treinamento desportivo, $3^{a}$ ed, São Paulo: Ibrasa, 1987,

WEINECK, J, Biologia do Esporte, São Paulo: Manole, 1991, 